\title{
Risk-based recall intervals recommended
}

\begin{abstract}
National Institute for Clinical Excellence (NICE). Dental recall: recall interval between routine dental examinations (www.nice. org.uk/page.aspx?o = 225866)

This summary of clinical guideline 19 from NICE includes recommendations for patients of all ages (both dentate and edentulous patients) and covers primary care received from NHS dental staff (dentists, independent contractors contracting within the NHS, dental hygienists and therapists) practising in England and Wales. The guideline takes into account the potential of the patient and the dental team to improve or maintain the quality of life and to reduce morbidity associated with oral and dental disease. The guideline does not cover intervals between dental examinations that are not routine dental recalls; that is, intervals between examinations related to ongoing courses of treatment, or part of current dental interventions, nor does it cover emergency dental interventions, or intervals between episodes of specialist care.
\end{abstract}

\section{Guidance}

The recommended interval between oral health reviews should be determined specifically for each patient, and tailored to meet his or her needs, on the basis of an assessment of disease levels and risk of or from dental disease.

This assessment should integrate the evidence presented in this guideline with the clinical judgement and expertise of the dental team, and should be discussed with the patient.

During an oral health review, the dental team (led by the dentist) should ensure that comprehensive histories are taken (see Table 1), examinations are conducted and initial preventive advice is given. This will allow the dental team and the patient (and/or his or her parent, guardian or carer) to discuss, where appropriate:

- the effects of oral hygiene, diet, fluoride use, tobacco and alcohol on oral health;

- the risk factors (see Table 1) that may influence the patient's oral health, and their implications for deciding the appropriate recall interval;

- the outcome of previous care episodes and the suitability of previously recommended intervals;

- the patient's ability or desire to visit the dentist at the recommended interval;

- the financial costs to the patient of having the oral health review and any subsequent treatments.

The interval before the next oral health review should be chosen, either at the end of an oral health review if no further treatment is indicated, or on completion of a specific treatment journey.

The recommended shortest and longest intervals between oral health reviews are as follows:

- The shortest interval between oral health reviews for all patients should be 3 months.

- The longest interval between oral health reviews for patients younger than 18 years should be 12 months.

- The longest interval between oral health reviews for patients aged 18 years and older should be 24 months.

For practical reasons, the patient should be assigned a recall interval of $3,6,9$ or 12 months if he or she is younger than 18 years,

Address for correspondence: National Institute for Clinical Excellence, MidCity Place, 71 High Holborn, London WC1V 6NA, UK. or $3,6,9,12,15,18,21$ or 24 months if he or she is aged 18 years or more.

The dentist should discuss the recommended recall interval with the patient and record this interval, and the patient's agreement or disagreement with it, in the current record-keeping system.

The recall interval should be reviewed again at the next oral health review, in order to learn from the patient's responses to the oral care provided and the health outcomes achieved. This feedback and the findings of the oral health review should be used to adjust the next recall interval chosen. Patients should be informed that their recommended recall interval may vary over time.

\section{Commentary}

The UK National Health Service (NHS) guideline on dental recall intervals represents a milestone in the profession's continuing efforts to rationalise the practice of dentistry. This guideline takes aim at the 6-month recall dental shibboleth, which is anecdotally reported to have been popularised originally by dentifrice advertising in the late $1940 \mathrm{~s}$. This recall frequency has been so widely adopted that large proportions of both the public and profession regard the interval as almost sacrosanct, which has obvious implications for the adoption of the guideline.

From a purely procedural point of view, the Guideline Development Group have met most expectations for good practice. An existing systematic review of the literature pertaining to routine dental examinations was updated, and extensive consideration was paid to other published information on risk for oral disease, through the use of both existing systematic reviews and other high-quality evidence.

All subsequent recommendations are clearly labelled with the level of the supporting evidence. If criticism is warranted, it would be only for the absence of more information about how the recommendations were formulated from the meagre evidence in the literature and the opinions of experts. Although consensusbased methods are mentioned, some further detail on process would be welcome because so much of the recommendations is based on expert opinion.

Perhaps the most innovative aspect of this attempt to wean the profession from biannual dental examinations is the decoupling of the recall examination from other activities that frequently occur at the same appointment, namely radiographs and prophylaxes. Prophylaxis (scale and polish) is an especially contentious issue because, for some portion of patients, a 6-month 'cleaning' is perceived as both mandatory with respect to disease prevention, desirable with respect to appearance, and an entitlement if someone other than the patient is paying for it. By removing these considerations from those related purely to a recall examination, the emotionally charged atmosphere of denial will be considerably lessened when longer intervals for examination are proposed to some patients. Anticipating the results of the Cochrane review, this might be considered a 'divide and conquer' strategy, as it is probable that cost-effective prophylaxis frequencies will also eventually be shown to be related to risk.

The recommendations are straightforward, and for those familiar with the literature concerning risk assessment, it comes as no surprise that virtually all of the evidence grades are either ' $G G P^{\prime}$ (good practice point - based on clinical experience of the Guideline Development Group) or ' $\mathrm{D}$ ' (supported by case-control 
Table 1. Risk factors.

Medical history

Conditions where dental disease could put the patient's general health at increased risk (eg, cardiovascular disease, bleeding disorders, immunosuppression)

Conditions that increase a patient's risk of developing dental disease (eg, diabetes, xerostomia) Conditions that may complicate dental treatment or the patient's ability to maintain their oral health (eg, special needs, anxious/nervous/phobic conditions)

Social history High caries levels in mother and siblings

Tobacco use

Excessive alcohol use

Family history of chronic or aggressive (early onset/juvenile) periodontitis

Dietary habits

Exposure to fluoride

High and/or frequent sugar intake

High and/or frequent dietary acid intake

Use of fluoride toothpaste

Other sources of fluoride (eg, the patient lives in a water-fluoridated area)

Clinical evidence and dental history

Recent and previous caries experience

New lesions since last check-up

Anterior caries or restorations

Premature extractions because of caries

Past root caries or large number of exposed roots

Heavily restored dentition

Recent and previous periodontal disease experience

Previous history of periodontal disease

Evidence of gingivitis

Presence of periodontal pockets (BPE code 3 or 4) and/or bleeding on probing

Presence of furcation involvements or advanced attachment loss (BPE code *)

Mucosal lesions

Mucosal lesion present

Poor level of oral hygiene

Plaque-retaining factors (such as orthodontic appliances)

Low saliva flow rate

Saliva

Clinical evidence of tooth wear

Erosion and tooth surface loss

${ }^{a}$ BPE (Basic Periodontal Examination) code * is used when attachment loss is $\geqslant 7 \mathrm{~mm}$ and/or furcation involvements are present.

or cohort studies, case reports and expert opinion). It should be an embarrassment to the profession that the evidence guiding the selection of a frequency for the quintessential interaction with the patient is so weak.

Surprisingly, one aspect of the recommendations seems to perpetuate the professional mindset that has supported the inviolability of the 6-month recall interval. Only intervals divisible by three are recommended for use. Apparently, intervals of 4, 5, 7, $8,10,11,13,14,16,17,19,20,22$ and 23 months are unacceptable, although only practicality is cited in support of this proscription.

With over 60 years of inertia behind the 6-month recall examination interval, it is likely that strong measures will be required to effect substantial change among many practitioners. The guideline document includes two features that may help facilitate the transition.

One is the recommendation that practitioners discuss directly the selection of the recall interval with their patients each time a recall examination is completed. Simply involving the patient in this activity will enhance consideration of an individual patient's risk factors, since they will need to be cited by the practitioner in explanation of whatever interval is recommended. This formal attention to risk factors for oral disease, as opposed to the informal integration of this information that is assumed to take place whenever a clinician assesses a patient, may lead to more rational selection of recall intervals.

The other feature of the guideline that may aid adoption is the implementation section. The section presents a checklist with accompanying explanatory tables and a series of clinical scenarios. The checklist of "modifying factors" is suggested for evaluation at each oral health assessment. Unlike many other similar lists, this checklist does not attempt to weight the relative importance of the presence of any particular factor, and does not suggest a level or intensity of a factor that denotes elevated risk.

This lack of 'cookbook' risk assessment will undoubtedly deter some clinicians used to explicit step-by-step directions. It is a more honest approach, however, given the state of knowledge about the predictive power of individual risk factors and the validation status of such risk assessment recipes. Not only that, but it may also be more reassuring to the portion of the profession that fears the loss of individual autonomy in decision-making. The tables accompanying the risk factor checklist do a creditable job of summarising what is known about each risk factor, so that some guidance is offered for those completely at sea.

The entries in the checklist are not exhaustive. For example, the presence of implants or fixed and removable prostheses is not mentioned as a risk factor for caries, periodontal nor mucosal disease. Likewise, specific risk periods for caries, such as eruption of second molars when specific preventive procedures may appropriate, are not discussed. Nevertheless, the checklist is sufficient to introduce naive clinicians to the concept of risk-based examination intervals. One feature that might be incorporated in future versions of the guideline, or provided at a NHS website for practitioners, would be a list of web- and paper-based sources for more information on risk assessment that is oriented towards dental team members.

The set of clinical scenarios that are also included in the implementation section arguably may be more useful than the checklist for some. These scenarios describe the salient facts for a specific patient, suggest a recall interval and provide a rationale for that selection. If practitioners choose to review these examples, virtually all of the main recommendations and discussion of risk factors will be reinforced.

The emphasis placed on oral cancer assessment in the examples is refreshing. Often, virtually all of the discussion of risk assessment is focused narrowly on dental caries or periodontal disease. The importance of periodic assessments of the oral tissues for premalignant changes and malignancies as a part of the periodic oral health assessment may not be appreciated by some clinicians. Featuring patient examples with elevated risk factors for oral cancer may be a potent reminder of professional responsibility.

A part of the remit for the guideline was to assess the costeffectiveness of risk-based intervals for dental recall examinations. 
The development group ultimately was unable to construct a model that would permit accurate assessment of the relative costeffectiveness of differing recall intervals applied population-wide, let alone differing intervals applied for subgroups of the population with differing risk profiles for oral disease. This result, however, should not mask the incremental progress contributed by the group's Markov model for progression of dental caries. The analysis (presented in Appendix E of the full report) improves on existing models both in the choice of outcomes, and in the selection of data upon which to base the estimates.

In summary, the guideline on dental recall is a well-constructed document. It is thorough in extracting available evidence from the literature, in supplementing that evidence with consensus opinions from an expert panel, and applying this information to answer (to the extent possible) a series of key clinical questions that address the effectiveness of risk-based examination intervals.

The recommendations formulated as a result of this process are logical, labelled in relation to the sources of the supporting evidence, and presented in a manner designed to facilitate application by practitioners. The guideline identifies audit criteria and suggests routine assessment of adherence as well as acceptability of the recommended behaviours, and urges development of information technology systems to help these audits at several levels. One can hope that these audits will be performed, and that the results will be thoroughly analysed.

The responses of dentists and their patients could be a harbinger of the extent to which evidence-based guidelines will alter practice. This is an important guideline that focuses on a core function performed by dentists. Its reception by the profession in terms of the speed and breadth of adoption within the NHS should offer some indication of the future of voluntary as opposed to administratively imposed changes to practice style to enhance clinical- and cost-effectiveness.

To generalise from the experience with this particular model when considering adoption of all evidence-based guidelines may lead to a biased estimate, however. Although intellectually appealing, the decoupling of the examination and prophylaxis intervals will certainly be a source of frustration, if not outright anger, for many practitioners and patients alike. Patients electing to maintain a 6-month interval for cleaning may be obliged to make more visits to the dentist to receive the same services prior to initiation of the guideline, and patients who alter their cleaning intervals to match lengthened examination schedules may feel short-changed. Because it was not part of the remit, the implementation section offers no guidance to practitioners for strategies that might help attenuate these predictable perceived injustices. Only if the systematic review of prophylaxis frequency finds that oral health is relatively insensitive to the intervals between scaling and cleaning could a scientific case be made for recoupling the examination and cleaning services for low-risk individuals.

\section{Jim Bader \\ Department of Operative Dentistry, School of Dentistry, University of North Carolina, Chapel Hill, NC 27599-7450, USA}

Evidence-Based Dentistry (2005) 6, 2-4.

doi:10.1038/sj.ebd.6400305 